\title{
Evaluation of First Electronic Case Reports received in Illinois
}

\section{Stacey Hoferka}

Illinois Department of Public Health, Chicago, Illinois, United States

Objective

Comparison of content in eCR and ELR cases reporting Review technical challenges and strategies for data management

Introduction

Communicable disease reporting from providers can be a time-consuming process that results in delayed or incomplete reporting of infectious diseases, limiting public health's ability to respond quickly to prevent or control disease. The recent development of an HL7 standard for automated Electronic initial case reports (eICR) represents an important advancement for public health surveillance. The Illinois Department of Public Health (IDPH) participated in a pilot with the Public Health Informatics Institute and an Illinois-based provider group to accept eICR reports for Gonorrhea and Chlamydia.

\section{Methods}

The provider group working with their EHR vendor submitted a batch of CT and GC reports directly to IDPH in September 2017 according to the published eICR standard. A summary of the provider and PHII work has been presented previously in the STI eCR Learning Community. The eICR reports received from the provider were compared to case report data in the communicable disease surveillance system, I-NEDSS. Data was extracted from I-NEDSS that included race and ethnicity, timing of specimen collection, result, ELR submission surveillance action and treatment.

\section{Results}

IDPH received a batch of 89 files containing 77 unique persons, with 54 chlamydia (CT), 13 Gonorrhea (GC) and 10 co-infected case reports. The communicable disease surveillance system had captured 76 (98.7\%) of the persons reported in the pilot. Among those, an Electronic Laboratory Report (ELR) was received for 72 (95\%) cases, on average within 1 day of the lab report date. Data in I-NEDSS had a completion of $45 \%$ for race and ethnicity compared to $99 \%$ for race and $92 \%$ for ethnicity in the eICR files. Information on treatment in the surveillance system was reported for 18 (24\%) cases compared to 67 (87\%) cases.

\section{Conclusions}

This pilot was the first submission of real patient data submitted using the eICR standard to IDPH. Data was more complete from provider eICR reports for key demographic of race and ethnicity and treatment. A comparison with the current surveillance system showed near complete and timely case capture from ELR data. Integrated reporting of both ELR and eICR can produce a more complete case report through automated submissions and potentially reduce burden of data collection on health department communicable disease investigators. As public health reporting moves in this direction, public health agencies will have some substantial tasks to correctly ingest, map and interpret the increased amounts of information that are contained in the eICR. Further, the advantages of case reporting will be dependent on automated processes within the communicable disease system to merge data and apply business rules to automatically process completed case reports for high volume diseases, such as STIs. This work will continue as providers are ready to submit reports from different vendor products from a near real-time production environment.

ISDS Annual Conference Proceedings 2019. This is an Open Access article distributed under the terms of the Creative Commons AttributionNoncommercial 4.0 Unported License (http://creativecommons.org/licenses/by-nc/3.0/), permitting all non-commercial use, distribution, and reproduction in any medium, provided the original work is properly cited. 